Revue d'histoire de l'Amérique française

REVUE D.HISTOIRE DE L'AMÉRIQUE FRANÇAISE

\title{
Une entreprise maritime canadienne-française - la Compagnie du Richelieu, 1845-1854
}

\section{Gerald Tulchinsky}

Volume 26, numéro 4, mars 1973

URI : https://id.erudit.org/iderudit/303212ar

DOI : https://doi.org/10.7202/303212ar

Aller au sommaire du numéro

Éditeur(s)

Institut d'histoire de l'Amérique française

ISSN

0035-2357 (imprimé)

1492-1383 (numérique)

Découvrir la revue

Citer cet article

Tulchinsky, G. (1973). Une entreprise maritime canadienne-française — la Compagnie du Richelieu, 1845-1854. Revue d'histoire de l'Amérique française, 26(4), 559-582. https://doi.org/10.7202/303212ar d'utilisation que vous pouvez consulter en ligne.

https://apropos.erudit.org/fr/usagers/politique-dutilisation/ 


\title{
UNE ENTREPRISE MARITIME CANADIENNE-FRANÇAISE LA COMPAGNIE DU RICHELIEU, 1845-1854
}

\author{
Gerald Tulchinsky \\ Département d'histoire \\ Queen's University
}

I

Si le commerce maritime du Saint-Laurent, en amont de Montréal, fut contrôlé par de grandes compagnies dont les propriétaires étaient tous anglais, les Molson, les Torrance et les Tate, le commerce de la partie du fleuve entre Montréal et Trois-Rivières et de la vallée du Richelieu intéressa, cependant, de petites compagnies dont les membres étaient, pour la plupart, canadiens-français. Et bien qu'il y eût dans ces régions de sérieux reculs dans la culture du blé au début du XIX ${ }^{e}$ siècle, et qu'une crise agricole se développa au milieu des années $1830^{1}$, il y avait encore, dans des villages de la rive sud du Saint-Laurent et le long du Richelieu, des groupes de petits entrepreneurs canadiens-français actifs et possédant du capital. ${ }^{2}$ Prévoyants et ingénieux, ils furent attirés durant les années 1830 par l'aventure de la navigation commerciale par bateaux à voile et à vapeur. Leur activité s'accrut au début des années 40 stimulée probablement par l'achèvement du canal de Chambly qui facilitait l'exportation de plus en plus considérable du bois vers le marché américain. L'usage plus répandu des bateaux à vapeur sur les voies navigables canadiennes et la croissance générale de l'activité économique, due à la construction de chemins de fer, favorisèrent le commerce maritime le long du Richelieu, de Montréal au lac Champlain.

Durant cette période un certain nombre d'hommes d'affaires canadiens-français de Montréal devinrent aussi entrepre-

1 Fernand Ouellet, Histoire économique et sociale de Québec, 17601850 (Montréal, 1966).

2 Voir Alex. Jodoin et J.-L. Vincent, Histoire de Longueuil et de la Famille de Longueuil (Montréal, 1889), chap. 23 et A. Couillard-Després, Histoire de la Famille et de la Seigneurie de Saint-Ours - La Famille et la Paroisse de Saint-Ours, 1785-1916 (2 vol., Montréal, 1917), II: 402-403. 
neurs dans le commerce maritime; en 1845 les deux groupes, l'un de la métropole et l'autre de la région, se réunirent pour créer la Société de Navigation du Richelieu, le premier ancêtre de Canada Steamship Lines ${ }^{3}$. Nous étudierons ici, à travers ses procès-verbaux, cette société qui, en 1847, reprend le nom de la Compagnie du Richelieu et qui devient l'entreprise maritime la plus importante et probablement la plus rentable de la région. Ce fut autour d'elle que se concentrèrent les efforts qui réussirent pendant quelque temps à grouper, en une seule société, les diverses compagnies de bateaux à vapeur qui desservaient le Richelieu et le Saint-Laurent. Les propriétaires de ces bateaux se rendaient de plus en plus compte qu'une réduction de la concurrence les aiderait à maintenir leurs profits face à la rivalité des chemins de fer. Dès 1853 prit fin l'entente conclue, trois ans plus tôt, entre la Compagnie et les autres propriétaires importants de bateaux à vapeur du Richelieu, dans le but de limiter la concurrence. La Compagnie se retrouva alors devant la nécessité de décider si elle devait conclure des arrangements avec ses rivaux, se risquer à nouveau à la rivalité ouverte et à une ruine probable, ou se retirer tout à fait du commerce du Richelieu. Elle décida de se retirer.

La Compagnie du Richelieu, malgré son nom, ne borna pas son activité à cette rivière. Après 1850 la firme commença lentement à prendre part au commerce maritime du Saint-Laurent pour répondre aux besoins de l'expansion du fret, du transport des passagers et pour remorquer radeaux et chalands entre Montréal, les autres endroits de la rive du Saint-Laurent et le Richelieu. Ce transfert vers le Saint-Laurent a dû être complété vers la fin de la saison maritime de 1853, car après cette année, la Compagnie n'envoya plus ses bateaux sur le Richelieu. Même après la formation du cartel des propriétaires de bateaux en 1850 , les profits furent modiques car le volume du commerce maritime sur le Richelieu diminua sensiblement chaque année. La décision de se retirer du Richelieu et d'entrer dans le commerce du Saint-Laurent, prise par la Compagnie en 1853, fut moins une poussée vers de nouvelles frontières maritimes que l'abandon d'une voie commerciale dont l'importance déclinait sans cesse. L'année 1853 est donc une année charnière. Elle marque, pour une compagnie de grandeur moyenne, forte de huit années d'expérience dans un domaine où la concurrence était acharnée, le retrait du Richelieu et l'entrée dans le Saint-Laurent entre Québec et Montréal. Cette

${ }^{3}$ William Henry Atherton, Montreal, 1535-1914 (3 vol., Montréal, 1914), II : 577. 
décision est importante car la Compagnie, quoique plus petite que les lignes Molson et Torrance qui dominaient le commerce entre les villes du bas Saint-Laurent, fut en mesure de leur disputer une part de la circulation fluviale entre Montréal et Québec après 1854. Ainsi, la croissance de la Compagnie jusquelà montre la capacité d'un groupe d'hommes d'affaires canadiensfrançais à monter une entreprise. En outre, l'entrée de la Compagnie du Richelieu dans le Saint-Laurent, ainsi que la puissance croissante au sein de la Compagnie d'un groupe de Montréalais, illustrent le contrôle de plus en plus complet que prenait Montréal du transport maritime.

\section{II}

La rivière Richelieu, comme le signale le professeur Lower, était depuis 1827 une importante voie commerciale pour l'exportation du bois canadien aux Etats-Unis ${ }^{4}$. Vers la fin des années 1830 et au début des années 1840 , la valeur des exportations qui passaient par la ville de Saint-Jean, atteignait des chiffres importants. C'était à Montréal qu'était entreposée la plus grande partie du bois destiné à ce marché américain. Bien que Montréal fût secondaire par rapport à Québec comme centre d'exportation du bois, les produits forestiers n'en représentaient pas moins un élément majeur de son commerce ${ }^{5}$. Au début de la décennie 1830-1840, on trouvait, aux environs de Montréal, de nombreuses scieries qui transformaient le bois nécessaire à la construction de maisons et de bateaux ${ }^{6}$. A Chambly, situé à la tête du canal, on préparait beaucoup de bois pour le marché américain. ${ }^{7}$

Stimulées par ces conditions favorables pendant les années 1830 et 1840 , de grandes et petites compagnies maritimes furent créées pour assurer le trafic, de et vers Montréal, le long du Saint-Laurent et du Richelieu ${ }^{8}$. C'était déjà une coutume éta-

${ }^{4}$ Arthur R. M. Lower, The North American Assault on the Canadian Forest: A History of the Lumber Trade Between Canada and the United States (Toronto, 1938), 97.

5 H. A. Innis et A. R. M. Lower, Select Documents in Canadian Economic History, 1783-1885 (Toronto, 1933), 247.

6 Michael S. Cross, "The Dark Druidical Groves, the Timber Community of British North America to 1854" (thèse manuscrite de Ph.D., Université de Toronto, 1968), 148-149.

7 Keith A. Parker, "The Staple Industries and Canadian Economic Development, 1841-1867" (thèse manuscrite de Ph.D., Université du Maryland, 1966), 63.

8 Couillard-Després fournit la liste des bateaux à vapeur en service sur le Richelieu au milieu du XIXe siècle, op. cit., II : 402. 
blie pour plusieurs entrepreneurs de former une compagnie afin d'acheter un bateau à vapeur déjà construit ou d'en commander un neuf. Dès 1832, suivant l'exemple d'une expérience similaire remontant à $1825^{\circ}$, un groupe d'investisseurs de la région établirent la Ferry Boat Company, sous la direction de S. H. Carlou, du pilote montréalais Flavien Amelin [Hamelin] et de Paul Kauntz, dans le but de posséder et de piloter le Canadian Patriot, bateau à vapeur de 196 tonnes, pour le service traversier entre Montréal et Longueuil ${ }^{10}$. Deux ans plus tard, un autre groupe qui s'appelait la "Source de Varennes Steamboat Company" fit bâtir un plus gros vaisseau destiné au commerce du Saint-Laurent. Paul et Pierre-Eustache Lussier et François-Antoine La Rocque furent chargés de diriger les opérations ${ }^{11}$. Pendant la même année, l' "Union canadienne Joint Stock Company" fit construire le bateau à vapeur Union Canadienne, entreprise où figurent les noms d'Andrew Achim, de Victor Choiny et de Joseph Vincent, tous de Longueuil ${ }^{12}$.

Les trois compagnies avaient le même type d'organisation. Chacune possédait soixante-quatre actions, dont la plupart étaient détenues, en nombre d'une ou deux actions, par des actionnaires dont on ignore l'identité. En outre, chaque compagnie était dirigée par des fiduciaires ou par des directeurs qui possédaient le plus grand nombre d'actions. Ces trois compagnies, dont les directeurs et vraisemblablement la plupart des actionnaires étaient des Canadiens français, se ressemblaient également par leur intérêt dans le commerce entre Montréal et Longueuil. ${ }^{13} \mathrm{Si}$ l'on fait exception de François-Antoine La Rocque, puissant marchand montréalais et ancien négociant de fourrures, les propriétaires de ces vaisseaux semblent avoir été d'une importance économique plutôt moindre dans la région.

La plupart des promoteurs de ces entreprises devaient être de la classe des propriétaires de bateaux, des capitaines et des copropriétaires de petits voiliers qui naviguaient sur le fleuve. Les activités de ces entrepreneurs sernblent avoir connu une

9 Jodoin et Vincent, op. cit., 548.

10 Archives publiques du Canada, R.G. 12, Département du Transport A, Département de la Marine, Registres maritimes, CLXXV: 3.

11 Ibid.: 8.

12 Ibid.: 13; A. Leblond de Brumath, Histoire populaire de Montréal, depuis son origine jusqu'à nos jours (Montréal, 1890), 364 .

13 Ce commerce a pu attirer d'autres entrepreneurs canadiens-français. Jodoin et Vincent, op. cit., 550; et le "Champlain et St-Laurent" et le "St. Lawrence and Atlantic" ont plus tard mis en service leurs propres traversiers. 
période de croissance considérable dans les années 1830 et 1840 , alors que la circulation, tant locale que directe (Montréal-TroisRivières-Québec), augmenta. Les registres maritimes ${ }^{14}$ de Montréal indiquent une croissance considérable pendant ces décennies du nombre de petits bateaux construits pour la circulation fluviale et de leurs activités commerciales. ${ }^{15}$ Tandis que les grandes compagnies de bateaux à vapeur semblaient concentrer leurs opérations sur la circulation des passagers et du fret de valeur entre les grandes villes du fleuve, les propriétaires de sloops et de goélettes trouvaient le champ libre dans le domaine du transport entre les nombreux petits villages le long du SaintLaurent et du Richelieu. Le volume du trafic n'était sans doute pas assez considérable ni assez profitable pour des compagnies de bateaux à vapeur; il permettait pourtant une rémunération suffisante à ces capitaines-propriétaires de vaisseaux sans ligne régulière dont l'investissement et les frais étaient minimes.

Ce genre d'affaires qui, tout en n'offrant pas de possibilités de progrès spectaculaires, prospérait et s'élargissait de façon modeste, attirait par conséquent encore des investissements et trouvait une place sur le Saint-Laurent et sur le Richelieu. Si les propriétaires de ces vaisseaux étaient pour la plupart des Canadiens français, c'était probablement à cause de l'absence presque totale des Anglais dans les petits villages situés le long du fleuve, et à cause aussi de la forte orientation de la bourgeoisie commerçante de Montréal vers le commerce dans les régions de l'intérieur. Des marchands canadiens-français, comme Joseph Masson, entretenaient par contre des rapports commerciaux étroits avec ces villages le long du Saint-Laurent et du Richelieu ${ }^{16}$.

Ces entrepreneurs n'étaient pas tous des marins. Parmi les occupations des propriétaires actionnaires des bateaux immatriculés à Montréal au cours de ces années, inscrites dans les registres maritimes, on trouve, en 1837, comme étant les seuls propriétaires du bateau à vapeur Charlevoix, un sculpteur, un tanneur et un médecin ${ }^{17}$ : Victor Chenier, Henry Monjeau et Charles Boucher de Grosbois de la ville de Chambly. Ils faisaient pourtant exception à la règle, car ceux qui possédaient presque tous les voiliers construits et immatriculés à INontréal

14 Voir les Registres maritimes cités, CLXXIV, CLXXV : passim.

15 Couillard-Després, II : 402-403.

16 Université de Montréal, Collection Baby, boîte 124. Voir les dossiers A. Cartier, Joseph Cartier, Eustache Cartier.

17 Registres maritimes, CLXXV : 27. 
aux années 1840 étaient des marins s'inscrivant pour la plupart comme "maîtres-mariniers" ${ }^{18}$. Des quatorze goélettes et sloops de cinquante tonnes ou plus ainsi immatriculés entre 1841 et 1851, onze appartenaient à la classe des capitaines-propriétaires de vaisseaux ${ }^{19}$, et un à des constructeurs de bateaux ${ }^{20}$. Bien que naviguant le long du Saint-Laurent et du Richelieu, ils semblent avoir établi leur siège à Montréal. Le système commercial l'aurait exigé, car la majeure partie des affaires de ces commerçants se faisaient dans le domaine de l'exportation des matières premières et des produits manufacturés, ces derniers surtout devant passer par Montréal.

Même si ces entrepreneurs-marins faisaient bâtir le plus souvent des voiliers, des goélettes de cent tonnes ou des sloops de cinquante tonnes, ils possédaient néanmoins la capacité évidente de financer et de gérer des bateaux à vapeur. La formation des trois compagnies de bateaux-traversiers Montréal-Longueuil, aussi bien que la mise en service du Charlevoix en 1837 prouvent qu'une certaine puissance financière et administrative existait parmi les capitaines de vaisseaux canadiens-français et la petite bourgeoisie de la région. C'est ainsi qu'un groupe de ces personnes forma en 1845 la Société de Navigation du Richelieu pour faire construire et administrer les opérations du bateau à vapeur le Richelieu, et nous fournit un exemple de plus d'entreprise maritime dans cette région. On n'aurait pas pu prévoir, à l'origine, que cette entreprise connaîtrait succès et longue vie, mais le seul examen de son histoire depuis sa fondation jusqu'à sa transformation en grande compagnie de navigation sur le Saint-Laurent en 1853 fait voir une période de neuf années d'habile gestion.

\section{III}

Trente-cinq actionnaires en tout et partout participèrent à l'aventure menée par Jacques-Félix Sincennes, Augustin SaintLouis et Louis Saint-Louis, qui à eux seuls achetèrent soixante des cent trente actions ${ }^{21}$ de la Compagnie, actions dont la valeur était de vingt-cinq livres chacune. Les investisseurs étaient tous canadiens-français, à l'exception de cinq, et ils habitaient la plupart la vallée du Richelieu. Pierre-Edouard Leclerc, Victor

18 Ibid.: passim.

19 Ibid.: 41, 68, 80, 83, 84, 86, 92, 118, 122 ; CLXXVI : 17, 51.

20 Ibid., CLXXV : 158.

21 Canada Steamship Lines, Montréal, Société de Navigation du Richelieu, Livre des procès-verbaux, s.l. 
signataires le 7 octobre 1845 . L'on ne put beaucoup accomplir en cette fin de saison maritime de 1845 , mais la firme était en position de bien commencer le travail de l'année suivante en sollicitant des contrats avec des marchands établis le long de sa route et en annonçant ses services à venir. La saison de 1846 fut très occupée; à la fin de l'année il resta à la Compagnie un bon profit net de $£ 1295^{33}$, ce qui lui permit de payer un dividende de $£ 8$ sur chaque action. Ce qui donnait un rendement de trentedeux pour cent. Timothée Franchère, président de la Compagnie, minimisa cette réussite quand il qualifia les progrès de l'année de "satisfaisants" 34 .

L'année 1847 fut encore meilleure pour la Société de Navigation du Richelieu. Même si l'on n'ajouta aucun nouveau vaisseau, les profits augmentèrent considérablement. Malgré une hausse des frais d'opération, le profit net monta à plus de $£ 2000$. La Compagnie put payer $£ 14$, ou un rendement de cinquante-six pour cent à chaque actionnaire, tout en retenant une somme modeste en cas d'imprévu.

Il n'est donc pas étonnant que d'autres compagnies s'établirent pour exploiter le commerce de cette région. Une de ces firmes fut la Société de Navigation du Saint-Laurent et du Richelieu, créée en septembre 1847, avec un capital de $£ 6500$. Deux cent soixante actionnaires contribuèrent à mettre en service le Jacques Cartier, grand bateau à vapeur construit à Sorel. ${ }^{35} \mathrm{La}$ nouvelle compagnie comptait beaucoup de nouveaux actionnaires en plus de ceux qui participaient déjà à la firme aînée. Si cette dernière était détenue en bonne part par des Montréalais, la nouvelle Compagnie avait une proportion beaucoup plus élevée de participants provenant des villages du Saint-Laurent et du Richelieu: Saint-Denis, Saint-Artoine, Saint-Mathias, Saint-Ours, Saint-Simon, Saint-Charles, Chambly, Belœil, La Présentation, Berthier et Sorel. En fait, des Montréalais ne possédaient que trente actions dans cette nouvelle entreprise que dominèrent de gros actionnaires comme Jacques-Félix Sincennes et George Cairns, ayant chacun quarante actions alors que Timothée Franchère, P.-E. Leclerc et L. Guéront en possédaient chacun vingt. ${ }^{30}$

33 Canada Steamship Lines, Montréal. Procès-verbaux de la Compagnie du Richelieu, 15 février 1854, désormais cités comme "Procès-verbaux". Les procès-verbaux de la Compagnie pour cette date contiennent un sommaire précieux des reçus, des dépenses et des profits nets annuels entre 1846 et 1853.

34 Société de Navigation du Richelieu, Procès-verbaux du 15 fév. 1847.

35 Procès-verbaux, s.l.n.d.

36 Ibid. 
Le fait que ces hommes se distinguaient également dans la Compagnie plus ancienne peut sans doute expliquer les projets de l'unification des deux compagnies presque dès l'achèvement du bateau à vapeur de la nouvelle firme en 1848. En effet, à en juger par la façon rapide dont se produisit leur fusion, on peut croire qu'il y avait eu arrangement. Vite réalisée vers le début du mois de mars 1848 "sur des bases justes et équitables pour les deux Sociétés" ${ }^{37}$, cette incorporation prévoyait une division des profits au prorata de la valeur de chaque bateau, ou selon des proportions d'un tiers et de deux tiers aux propriétaires du Richelieu et du Jacques Cartier respectivement. Les deux bateaux à vapeur, administrés par une seule compagnie de façon à partager raisonnablement la circulation entre eux, offriraient un service plus efficace le long de la voie desservie. Ce fut la première des fusions à venir.

Il était heureux que cette nouvelle Compagnie du Pichelieu (ainsi désignée depuis la fusion des deux groupes), grâce à deux bateaux à vapeur et à son plus grand nombre d'actionnaires, ait la force d'affronter de nouveaux concurrents. Déjà pendant la saison maritime de 1848, elle dut faire face à la rivalité sérieuse de quelques autres bateaux à vapeur alors que la menaçait une concurrence encore plus forte de la part du chemin de fer "Champlain et Saint-Laurent" que ses propriétaires projetaient de prolonger vers le sud depuis Saint-Jean jusqu'à la frontière américaine. De toute manière, le rendement de la saison 1848 se ressentit de la rivalité des autres bateaux. Les profits de la Compagnie tombèrent à moins de la moitié du chiffre de 1847 et il y eut une baisse proportionnelle des dividendes. Ainsi, lors de la réunion annuelle des actionnaires qui eut lieu vers la fin de février 1849, il fallut bien envisager la dure réalité: non seulement les profits avaient considérablement baissé, mais l'avenir présageait une concurrence accrue. Louis Marchand, commerçant montréalais, ancien conseiller muricipal et membre de la Commission du port ${ }^{38}$, signala que le Jacques Cartier avait coûté presque $£ 400$ de plus qu'on avait pu toucher par la vente des actions, et qu'en conséquence, il faudrait trouver du capital supplémentaire. ${ }^{39}$

Durant la saison maritime de 1849 , les effets de la concurrence réduisirent encore davantage les profits de la Compagnie.

37 Ibid., le 10 mars 1843.

38 Liste préliminaire manuscrite des hommes d'affaires de Québec, 1851-1900, préparée par l'équipe du Dictionnaire biographique du Canada; National Harbours Board, Montreal, Trinity Letter Books, CLVI, Benjamin Holmes à Louis Marchand, 28 mai 1852.

39 Procès-verbaux du 27 fév. 1849. 
A cause de "l'opposition acharnée contre laquelle nous avons eu à lutter... nous avons été dans la nécessité de transporter les passagers de pont à Sorel, Berthier et autres ports intermédiaires à Montréal pour la modique somme de dix sols et les contre-produits et effets à des taux très réduits...,", rapportet-on dans les Minutes. ${ }^{40}$ On ne manqua pas de rappeler aux actionnaires que la crise économique générale affectait également le commerce maritime par une baisse appréciable du volume des produits transportés. En outre, l'épidémie de choléra qui sévissait alors dans le Bas-Canada avait fait sentir ses effets dévastateurs sur la circulation du fret et des passagers ${ }^{41}$. Par conséquent les profits furent réduits à $£ 763 / 3 / 9$ et l'on ne paya qu'un petit dividende d'un peu plus de sept pour cent. Si, selon des critères modernes ce rendement peut paraître acceptable, il fut loin de réjouir des actionnaires qui avaient connu le haut rendement initial de la Compagnie en 1846 et 1847.

Ce qui empêcha surtout la Compagnie de continuer de réaliser de grands profits fut la rivalité croissante des autres compagnies de bateaux à vapeur qui faisaient le même commerce sur le Richelieu et le Saint-Laurent. On ne pouvait donc que souhaiter la réduction sinon l'élimination complète de cette concurrence. Une nouvelle fusion avec une firme importante forcerait bientôt les autres à céder. Les propriétaires indépendants de bateaux à vapeur sur le Richelieu seraient mal avisés de continuer seuls, car ils risquaient de succomber devant l'éventuelle concurrence encore plus forte des chemins de fer. L'année 1850 était donc une période propice pour les compagnies de bateaux à vapeur d'envisager la question de leurs profits à court terme et de leur viabilité à la longue, car le prolongement du "Champlain et Saint-Laurent" touchait à sa fin et la voie ferrée du "St. Lawrence and Atlantic" approchait de Sherbrooke. Ainsi, doublement vulnérables, les propriétaires de bateaux à vapeur de la région furent poussés à rechercher quelque forme de fusion.

Augustin Saint-Louis, marchand de Sorel, était associé à la Compagnie du Richelieu depuis 1845, année où il avait acheté, avec des parents, une vingtaine d'actions. Mais sa participation ne s'étendit jamais à la direction de la Compagnie et il est possible qu'il se soit tôt débarrassé de ses actions. Bien que l'affaire s'annonçait avantageuse, Saint-Louis s'objecta sans doute à

40 Ibid., 28 février 1850. La concurrence était si sévère sur le Richelieu que l'on dut réduire les tarifs à plusieurs reprises selon William Wood, All Afloat, $A$ Chronicle of Craft and Waterways (Toronto, 1915), 149 .

41 Procès-verbaux du 28 février 1849. 
certaines décisions de l'administration et à la position proéminente de Jacques-Félix Sincennes qui achetait les actions des autres chaque fois qu'il le pouvait, vraisemblablement avec l'intention de dominer la Compagnie ${ }^{42}$. Saint-Louis fut peut-être aussi intéressé à diriger son propre bateau à vapeur. Sa participation au Richelieu a pu lui donner le goût de ce genre d'affaires et en plus une certaine expérience. On ne lui connaît pas de possession de vaisseau avant son placement dans le Richelieu, mais au mois de novembre 1847, il acheta le bateau à vapeur Oregon, conjointement, semble-t-il, avec Thomas Stuart Mears ${ }^{43}$, marchand de bois montréalais qui paraît être demeuré un associé silencieux dans cette affaire. Vers le même temps à peu près, Saint-Louis acheta le bateau à vapeur Vulcan ${ }^{44}$, qu'il mit en service sur le Saint-Laurent et sur le Richelieu, ce qui consterna la Compagnie du Richelieu. Outre ces vaisseaux dont il avait le contrôle entier, Saint-Louis dirigeait son propre bateau, le StLouis, fort probablement dans les mêmes régions que les autres ${ }^{45}$. Ainsi, sa participation au transport maritime constitue à la fois une affaire d'envergure et une réussite dont la force de concurrence menaçait d'étouffer la Compagnie du Richelieu.

La réussite de Saint-Louis dans le transport maritime demeure sans explication. Il est probable qu'une grande partie de son commerce consista dans le transport du bois expédié par son associé Mears. Mais, quelle que fût la clé de sa réussite, ce qu'il importe de noter, c'est la menace qu'elle représenta pour la Compagnie du Richelieu. Lors de la réunion annuelle de cette dernière, en février 1850 , les actionnaires approuvèrent la proposition de nommer un comité pour rencontrer Saint-Louis afin de discuter des moyens convenables de mettre fin à leur opposition mutuelle. Ils exhortèrent la direction à prendre d'autres mesures pour améliorer la capacité de concur-

$42 \mathrm{Au}$ début du mois de février 1848, Sincennes, qui possédait déjà quarante actions, en acquérait soixante-sept sur un total de 130 pour le Richelieu. Procès-verbaux, liste des transferts d'actions, s.l.n.d.

43 Registres maritimes, CLXXV: 107. L'association probable avec Mears est suggéré par le fait que Saint-Louis lui a hypothéqué l'Oregon le jour même où il lui a acheté le vaisseau. Ce fut alors un procédé commercial compliqué mais assez fréquent pour les propriétaires de bateaux à vapeur sur le Saint-Laurent. Voir ibid.: passim.

${ }^{44} \mathrm{La}$ date de l'achat du Vulcan demeure incertaine. Construit à Brockville en 1841, ce bateau fut enregistré de nouveau à Montréal au mois d'août 1851, avec Saint-Louis comme propriétaire commanditaire. Cet enregistrement ne signifie pas nécessairement un transfert de propriété; Saint-Louis en fut probablement le propriétaire avant 1851. Voir ibid., CLXXVIII: 2.

45 Ibid., CLXXV:73. 
rence de la Compagnie qui avait reçu diverses suggestions de plusieurs résidents et clients de la vallée du Richelieu. Donnant suite à ces recommandations, la Compagnie alloua un bateau à vapeur uniquement pour le remorquage des radeaux et des bateaux destinés aux Etats-Unis, vraisemblablement dans le but de permettre une circulation rapide sur le Richelieu, afin d'éliminer les délais causés par les arrêts à divers endroits pour embarquer et débarquer les voyageurs. ${ }^{46} \mathrm{En}$ outrè, on annoncerait dans les journaux locaux, une échelle de taux fixes pour le remorquage des bateaux selon leur tonnage et leur capacité. Enfin, on s'efforcerait de convaincre le Bureau des Travaux publics de réduire les droits d'usage du canal Chambly au montant de celui des canaux du Saint-Laurent.

S'il fut son rival le plus important, Augustin Saint-Louis ne fut pas le seul concurrent de la Compagnie du Richelieu. Il fallut compter avec la concurrence de petits voiliers qui s'adonnaient sur le Richelieu au transport du fret pour leurs propriétaires ou pour d'autres hommes d'affaires de la région. ${ }^{47}$ Quelques bateaux à vapeur appartenant soit à des entrepreneurs locaux ou à des Montréalais continuèrent d'annoncer régulièrement des voyages pour le fret et les passagers. D'autres vaisseaux étaient réservés presque exclusivement au transport du bois entre Bytown et Whitehall, port de l'Etat de New York, situé au pied du lac Champlain. On a pu constater l'importance de ce commerce lorsque, en février 1850, Jacques-Félix Sincennes annonça qu'il avait acheté, avec William McNaughton, marchand et "maître marin" de Saint-Ours, le bateau à vapeur Lord Stanley et cinq barges, "... dans la vue de continuer la ligne de Transport de bois de sciage entre Bytown et Whitehall ..."48 Sincennes voulait informer ses collègues parce qu'il savait depuis quelques années “... [combien] cette association de ma part pouvait peut-être porter ombrages à quelques membres de notre compagnie..." 49 Mais il ne put s'empêcher d'éveiller les soupçons des actionnaires envers celui qui, tout en restant directeur de la Compagnie du Richelieu, était devenu l'un de ses rivaux. On gardait cependant espoir de neutraliser une partie de la concurrence la plus menaçante ou même de l'éliminer, s'il était possible de traiter avec Saint-Louis.

46 Procès-verbaux du 28 fév. 1850.

${ }^{47} \mathrm{~L}$ 'on retrouve une liste partielle de ces vaisseaux dans les registres maritimes. Voir ibid., CLXXV: passim.

48 Ibid.: 77. La transaction ne fut pas complétée avant le mois de décembre 1850 .

49 Procès-verbaux du 28 fév. 1850. 
Moins d'un mois plus tard, un comité spécial de la Compagnie réuni à Montréal reçut des suggestions d'association avec Saint-Louis qui s'était fait représenter par George-Etienne Cartier ${ }^{50}$. P.-E. Leclerc de Saint-Hyacinthe, président de la Compagnie du Richelieu, rendit compte d'un accord qui prévoyait la réunion de ses bateaux et de ceux de Saint-Louis à partir du début de la saison de $1850 . .^{51}$

L'accord comprit des provisions pour la mise en commun de la capacité totale de transport des deux firmes, comme l'avait déjà fait en 1848 les propriétaires du Jacques Cartier et du Richelieu. Les onze dix-huitièmes des profits de cette association mutuelle devaient aller à la Compagnie, tandis que le reste serait versé à Augustin Saint-Louis et à son fils, Augustin..$^{52}$ Cet accord de trois ans stipula également que la Compagnie du Richelieu devait prendre en main la direction des vaisseaux de Saint-Louis comme faisant partie de sa propre flotte. Cependant, l'on devait engager Augustin Saint-Louis fils ainsi que son frère Félix Saint-Louis comme capitaine des vaisseaux St-Louis et Richelieu à un salaire respectivement de $£ 150$ et de $£ 125$ par an..$^{53}$ Ainsi, pour les amener à renoncer à leur entreprise concurrente, la Compagnie du Richelieu accorda aux Saint-Louis une proportion fixe d'un peu plus d'un tiers des profits totaux de la nouvelle combinaison financière et des postes comme salariés de la Compagnie. Celle-ci, par contre, avait l'avantage de contrôler ce qui devait être la majeure partie de la capacité de transport par bateaux à vapeur entre Montréal et le lac Champlain. De ce fait, elle assumait la responsabilité et les frais additionnels de l'entretien de trois vaisseaux supplémentaires.

Pendant quelque temps au moins, le syndicat qui comprenait les cinq bateaux à vapeur résista à la tentation d'étendre ses intérêts dans le Saint-Laurent en refusant les arrangements proposés par les frères John et Thomas Ryan qui étaient engagés dans un transport maritime de grande envergure comme copropriétaires de la People's Line depuis 1844. Au mois de mars 1846, le Bureau des Postes avait accordé à leur firme le contrat de transport du courrier entre Montréal et Québec durant la saison maritime ${ }^{54}$. Mais depuis, les Ryan et leurs associés (pour la

50 Ibid., du 14 mars 1850.

51 Ibid., du 18 fév. 1851.

52 Ibid.

53 Ibid., du 27 fév. 1851.

54 Archives de la Cour Supérieure, Montréal, Greffe de H. Griffin, 3 mars 1846, 21185. 
plupart des marchands québécois) avaient eu peine à rivaliser avec le groupe plus puissant des bateaux Molson, Torrance, Tate et Munn. Ayant pu obtenir le contrat du transport du courrier pour la saison maritime de 1850, Ryan échoua dans sa tentative de s'assurer la coopération du groupe pour remplir ses obligations. Il demanda l'aide de la Compagnie du Richelieu.

Les directeurs de la Compagnie eurent la sagesse de décliner sa proposition du 9 mars 1850 , “... vues les circonstances actuel [sic] dans les Quelles se trouve la Compagnie du Richelieu ..." 55 S'aventurer sur le Saint-Laurent pour y rivaliser avec le puissant groupe de Molson et autres aurait été extrêmement risqué pour eux, même s'ils avaient pu éliminer la concurrence sur le Richelieu. Au mois de mars 1850, ils ne pouvaient être assurés de maintenir les accords récemment conclus avec les Saint-Louis. Mais la tentation d'étendre leurs activités sur le Saint-Laurent était assez forte pour faire l'objet d'une reconsidération subséquente.

Si la saison maritime de 1850 apporta quelque satisfaction à la Compagnie, elle n'en fut pas moins dans l'ensemble, décevante. En passant en revue les activités de l'année, J.-B.-E. Durocher, de Saint-Charles, fit savoir aux actionnaires que sur des profits bruts de $£ 3969 / 3 / 1$, il ne restait à la Compagnie que $£ 2425 / 11 / 10$, après déduction de la part due aux Saint-Louis. ${ }^{56}$ En tenant compte du fait que cette somme se répartissait sur un capital de $£ 18000$ (valeur des cinq bateaux à vapeur), il serait payé seulement un petit dividende de seize pour cent, et pour ce faire, la Compagnie devrait emprunter quelque £1 500.57 En fait, le dividende fut beaucoup plus élevé, soit environ vingt-trois pour cent, car l'acquisition des bateaux à vapeur des Saint-Louis n'avait rien coûté à la Compagnie (sauf une proportion fixe des profits annuels). Ainsi, à vrai dire, le capital sur lequel se répartissait le dividende était d'environ $£ 11000$, ce qui comprenait l'investissement total dans le Richelieu et le Jacques Cartier ainsi que quelques petites additions d'équipement.

Néanmoins, les profits du syndicat ne furent pas de l'ordre que l'on espérait et ce, pour plusieurs raisons. D'abord, les frais d'opération des cinq vaisseaux furent plus considérables que prévus, comme on le signala en passant aux actionnaires. ${ }^{58}$ En

55 Procès-verbaux du 15 mars 1850.

56 Ibid., du 11 fév. 1851.

57 Ibid., du 18 fév. 1851.

58 Ibid. 
outre, le rendement du transport des voyageurs de Montréal ne fut pas profitable, surtout à cause de la concurrence des autres bateaux à vapeur et des chemins de fer. Il y avait encore une autre raison pour expliquer les minces profits: l'engagement, par contrats, pour le transport du bois scié de Québec et de TroisRivières jusqu'au lac Champlain qui occasionna une dépense énorme pour louer des barges. ${ }^{59}$ Dans un effort manifeste d'éviter les frais élevés de location de barges, les directeurs furent autorisés d'en acheter cinq à un prix maximum de $£ 500$.

La fin de la saison de 1850 marqua un tournant dans l'histoire de la Compagnie du Richelieu. Il importe de souligner qu'elle avait mené pendant cinq ans une existence assez florissante dans le commerce maritime fort concurrencé du Richelieu. L'union temporaire mais efficace des bateaux de la Compagnie et de ceux des Saint-Louis lui avait permis de contrôler pendant deux ans la plus grande partie du commerce sur le Richelieu et sur le Saint-Laurent, de Montréal à Québec, en limitant les frais et en augmentant les profits. Le déplacement des bureaux de la Compagnie depuis le Richelieu jusqu'à Montréal, “l'endroit le plus convenable pour y réunir le plus grand nombre d'actionnaires ..." ${ }^{60}$, avait été une décision très opportune. Ce déplacement montre bien que le commerce le plus lucratif, à savoir le bois de sciage destiné au lac Champlain, était disponible à Montréal et à des endroits situés plus haut sur l'Outaouais ou sur le Saint-Laurent. De plus, il est évident que la plupart des actionnaires, même si leurs noms ne furent pas systématiquement enregistrés, se trouvaient à Montréal.

$\mathrm{Au}$ début de l'année 1851, la Compagnie du Richelieu avait atteint une étape de son développement et en même temps entrait dans une période de transition. Elle n'était plus une entreprise purement locale où, à ses débuts, l'on vendait, dans les petits villages du Richelieu des souscriptions pour le Richelieu. Elle avait été absorbée par la métropole, tout comme les économies fluviales du Richelieu et du Saint-Laurent s'unirent à celles de Montréal par l'utilisation de plus en plus fréquente des bateaux à vapeur et des chemins de fer. Entre 1851 et 1853, le passage du Richelieu au Saint-Laurent et de l'entreprise locale au contrôle métropolitain se poursuivit. Il fut complété avant la fin de la saison de 1853.

50 Ibid.

60 Ibid. 


\section{IV}

Entre 1850 et 1854 , le volume du transport maritime sur le Richelieu baissait d'une manière alarmante. Bien qu'approximatifs, les chiffres de la circulation sur le canal de Chambly nous donnent quelques indications du volume sur le Richelieu aux années 1848 à $1854 .^{61}$ Entre 1848 et 1849 l'on remarque une hausse de presque 400 pour cent dans le tonnage des marchandises transportées sur le canal, soit de 18,835 à 77,216 tonnes, tandis que celui des vaisseaux augmente de presque 600 pour cent. ${ }^{62}$ Le trafic sur le canal continua à monter durant l'année 1850, mais le taux d'augmentation du tonnage des marchandises transportées et des vaisseaux fut beaucoup moindre que celui de l'année précédente, passant de 41.2 à 11.3 pour cent. Durant les quatre années qui suivirent, il n'y eut point d'augmentation importante dans le volume des marchandises transportées; mais pour l'année 1852, l'on constate une baisse de 20.9 pour cent. En même temps le tonnage total des vaisseaux baissa perceptiblement de 143,194 tonnes en 1850 à 90,691 tonnes en 1851 et à 82,618 tonnes en 1852 . Bien qu'en 1853 , le tonnage et des vaisseaux et des marchandises dépassât celui de 1852 , cependant il ne surpassa pas le niveau des années 1849-1850. ${ }^{63}$

Ceux qui s'attendaient au maintien du renouveau du commerce amorcé en 1853 furent déçus par la baisse massive d'activité pendant la saison de 1854 . Le tonnage des marchandises transportées sur le canal diminua de 27.6 pour cent, tandis que la capacité des vaisseaux baissa de 34.6 pour cent. ${ }^{64}$ Le déclin de l'industrie maritime du Richelieu, tel qu'indiqué par la circulation sur le canal de Chambly, ne fut pas suivi d'un déclin similaire du transport maritime sur les autres principales voies

61 Il est difficile, sinon impossible, de déterminer avec précision le volume du trafic entre les ports du St-Laurent et du Richelieu ainsi que la place qu'occupa Montréal dans ce commerce. Les statistiques disponibles sur le Richelieu ne nous renseignent que sur le trafic du canal de Chambly et du port de St-Jean; elles ne nous apprennent rien sur le transport des passagers et des marchandises entre les villages du Richelieu et la ville de Montréal. Les dossiers des compagnies maritimes qui contenaient ces renseignements précieux ont disparu. Pour des indications fort utiles sur l'exportation et l'importation à St-Jean aux années 1849,1850 et 1851 , voir I. D. Andrews, Report... on the Trade and Commerce of the British North American Colonies... (Washington, 1853), 427 et d'autres tables dans la Partie V. Toutes les indications sur le trafic dans les canaux ont été puisées dans les Tables of Trade and Navigation of the Province of Canada, for Year(s) 1850 (to 1854) (Québec, 1851-1855), tables 9 et 11. 62 Ibid. (1854), table 11.

63 Ibid.

64 Ibid. (1854), table 11. 
navigables au début des années 1850 . Les canaux du Saint-Laurent transportèrent un volume toujours plus grand de denrées et de bateaux après 1849. Le tonnage des denrées transportées en 1851 augmenta de 56.3 pour cent sur celui de 1850 et pour les années suivantes, l'on constata des augmentations de 9.3 (1852), de 32.2 (1853) et de 31.8 (1854) pour cent. ${ }^{65}$ La circulation sur le canal Welland, qui accusa une baisse de 15.3 pour cent en 1854 , avait augmenté chaque année depuis $1848 .{ }^{66}$ On peut dire la même chose et du canal de Burlington et de l'écluse Sainte-Anne sur l'Outaouais. ${ }^{67}$ Ainsi, le déclin du transport maritime sur le Richelieu contrastait avec cette croissance modérée, mais constante, du trafic sur les autres voies navigables.

Ce déclin se refléta également dans le cours des affaires à Saint-Jean où la valeur du commerce d'exportation et d'importation avec les Etats-Unis atteignit son sommet en 18501851 pour baisser abruptement dans les années suivantes. ${ }^{68} \mathrm{La}$ Compagnie du Richelieu et ses rivales se disputèrent un volume de transport qui diminuait sans cesse au début des années 1850, ce qui explique dans une large mesure le besoin urgent de consolidation puis l'obligation d'abandonner tout à fait le Richelieu.

$\mathrm{Au}$ cours des mois qui suivirent la réunion annuelle de février 1851, la Compagnie du Richelieu prit des décisions en vue de réduire les frais. Il fut décidé de maintenir la pratique de remorquer les radeaux pendant les voyages réguliers pour le transport des passagers, "en autant que la compagnie éprouverait des pertes considérables en discontinuant les towages (sic) ..." 69 Beaucoup plus important fut le problème du Richelieu, bateau vieillissant qui ne pouvait plus servir en même temps de remorqueur efficace et de bateau à passagers. Il fut décidé de ne plus l'affecter au remorquage, mais de le réserver au seul transport des voyageurs deux fois la sernaine, le long du Richelieu et entre Verchères, Varennes et Boucherville. ${ }^{70}$

Malgré cette mesure et celles déjà adoptées en vue d'améliorer l'efficacité des opérations de la Compagnie, l'année 1851 ne fut malheureusement pas plus profitable que celle qui l'avait précédée: l'on ne paya qu'un dividende de moins de quatorze pour cent, calculé sur la base d'un capital de $£ 16,000,{ }^{71}$ au lieu

65 Ibid. (1851-4), table 11.

66 Ibid. (1853), (1854), table 11.

67 Ibid.

68 Ouellet, op. cit., 616.

69 Procès-verbaux du 12 juillet 1850 .

70 Ibid., du 6 mai 1851.

71 Ibid., du 20 fév. 1852. 
de $£ 18,000$ à cause du retrait en fin de saison 1850 , du bateau à vapeur Vulcan appartenant à Saint-Louis. Bien que représentant un rendement réel plus élevé, puisque les bateaux des SaintLouis ne faisaient pas partie de l'investissement des actionnaires de la Compagnie, ce chiffre ne manqua pas toutefois de les décevoir. Il leur sembla qu'on n'atteindrait plus jamais le rendement des années 1846 et 1847. Les directeurs pouvaient entrevoir ce que leur réservait l'avenir.

Depuis plusieurs années, on retrouvait les mêmes hommes à la direction de la Compagnie. Et les actionnaires qui se réunirent en février 1851 continuèrent de maintenir en postes P.-E. Leclerc comme président, et Jacques-Félix Sincennes comme secrétaire-trésorier. Il y eut cependant quelques nouvelles nominations au conseil d'administration qui comptait maintenant quatre Montréalais. Leur présence reflétait l'orientation de la Compagnie vers la métropole.

Pendant les trois premières années, un seul Montréalais, N.-B. Desmarteau, avait fait partie du conseil d'administration. Il semblait être un négociant à l'aise et possédait deux magasins d'articles de nouveauté, l'un géré par lui-même et l'autre par Louis Marchand ${ }^{72}$. Desmarteau et Marchand ayant conjointement acheté douze actions dans le Jacques Cartier en 1848, se trouvaient donc parmi les plus gros actionnaires de la Compagnie. Marchand accéda au conseil en 1850, ainsi que J.-M. Lamothe, propriétaire à Montréal d'une librairie-papeterie, petite entreprise apparemment prospère. ${ }^{73}$ L'année suivante, on recruta encore deux Montréalais, Victor Hudon et J.-B. Boulanger. Boulanger était marchand tailleur ${ }^{74}$; Louis Marchand, qui avait été le partenaire de Desmarteau en $1845,{ }^{75}$ possédait, depuis 1851 , son propre commerce bien établi dans le même domaine, soit celui de l'épicerie et des articles de nouveauté. ${ }^{78} \mathrm{Ces}$ directeurs de la Compagnie étaient tous des hommes d'affaires peu considérables si l'on fait exception de Victor Hudon qui menait des affaires de plus grande importance. Ils n'en étaient pas moins tous prospères à la fin de la décennie 1840, et ils avaient l'expérience et les ressources suffisantes pour pouvoir étendre leurs intérêts d'entrepreneur au-delà des limites de leurs affaires de base à l'exemple de leurs concitoyens britanniques. Dans des

72 Montreal Directory (1845-46) : 61.

73 Lamothe n'a pas fait de publicité dans l'annuaire de McKay, mais

il a pu tenir une maison séparément de son magasin. Ibid., (1852): 137.

74 Ibid., (1852): 33.

75 Ibid. $(1845-46): 61,142$.

76 Ibid. (1852) : 169 . 
circonstances difficiles, ils avaient réussi à développer une entreprise de transport maritime d'une certaine importance et relativement prospère. Forts de cette réalisation et pouvant compter sur le dynamisme de leurs affaires respectives à Montréal, ils étaient préparés aux nouvelles perspectives des années 1850 qui leur offraient des possibilités séduisantes, mais aussi de cruelles déceptions.

C'est sans doute avec chagrin que les actionnaires apprirent que la famille Saint-Louis avait l'intention de retirer, à l'expiration du contrat, les navircs St-Louis et Vulcan. ${ }^{77}$ On ignore ce que les Saint-Louis entendaient faire de ces bateaux ainsi libérés, mais l'on prit la précaution d'exiger d'eux la protection des droits de la Compagnie en prévenant le danger de concurrence. Une nouvelle possibilité d'opérations commerciales s'ouvrit grâce à l'établissement de jonctions avec le chemin de fer "St. Lawrence and Industry Village" dont on avait complété la voie au mois de mai $1850 .^{78}$. C'était une courte ligne de douze milles entre Lanoraie, situé sur la rive nord du SaintLaurent à quelque cinquante milles en aval de Montréal, et le village d'Industrie (Joliette), situé sur la rivière L'Assomption dans le comté de Berthier. En 1852, on avait commencé à entrer en relations avec la Compagnie de chemin de fer, ${ }^{79}$ par l'intermédiaire de Jedediah Hubbell Dorwin, homme d'affaires montréalais et président du chemin de fer "Industry Village and Rawdon" qui se trouvait dans le prolongement vers le nord du "St. Lawrence and Industry Village", et qui était sur le point d'être achevé en 1852.80 En retour de $\$ 1,000$ en actions, la Compagnie de chemin de fer concéda à la Compagnie maritime "le privilège exclusif de faire accoster les vaisseaux à vapeur à l'exclusion de tous autres au quai de la dite Compagnie du Chemin de Fer... tant pour y prendre et débarquer seuls des voyageurs et des marchandises et effets transportés ou à être par le dit Chemin de fer" ${ }^{81}$. On s'entendit à l'avance pour l'établissement d'un service efficace entre les deux Compagnies.

77 Procès-verbaux du 20 fév. 1852.

78 Stats. Prov. Can. (1847), 10 \& 11 Vic. c. 64 ; Robert R. Brown, "The St. Lawrence and Industry Village Railway", Bulletin of the Railway and Locomotive Historical Society (1947) : 40.

79 Procès-verbaux du 20 fév. 1852.

80 Brown, op. cit., 40.

81 Procès-verbaux du 18 fév. 1853. 
L'année 1852 fut plus profitable pour la Compagnie et elle paya un dividende de vingt-cinq pour cent ${ }^{82}$. Mais malgré cette prometteuse montée des profits de la Compagnie, le retrait imminent des bateaux des Saint-Louis pouvait laisser présager un retour à une concurrence ouverte et incontrôlée à l'encontre de l'assurance donnée par les Saint-Louis. Les liens avec la compagnie de chemin de fer "St. Lawrence and Industry Village" ont pu être considérés par les actionnaires comme un moyen de compenser les profits réduits à cause d'une rivalité renouvelée. La perspective d'un monopole des affaires avec ce nouveau chemin de fer était séduisante. Bien que cette voie eût moins de trente milles de longueur, on avait en vue d'autres prolongements ${ }^{83}$ dans cette région à croissance rapide située sur la rive nord du Saint-Laurent. On destina le Jacques Cartier, le bateau à vapeur le plus récent et le plus efficace de la Compagnie, à naviguer sur le fleuve Saint-Laurent tandis qu'on laissa le vieux Richelieu sur la rivière Richelieu. Le transport par bateaux à vapeur sur cette rivière, autrefois très important, avait tellement diminué qu'à la fin de la saison maritime de 1853 l'on ne trouva plus profitable de desservir le Richelieu par bateau à vapeur.

"Vu la diminution du Commerce, transport de passagers etc. qui c'est (sic) opéré sur la Rivière Chambly [Richelieu] surtout depuis la Confection (sic) du chemin de fer du St-Laurent et de l'Atlantique ...84, la Compagnie décida de discontinuer les opérations du Richelieu et de vendre le vaisseau... pourvu que sa mise à prix ne soit pas moin (sic) de Deux mille quatre Louis Courant...". Un mois plus tard on fit savoir aux actionnaires que le Richelieu, vieux de huit ans, avait été vendu pour la somme de $£ 2500$ et que la Compagnie distribuerait le profit proportionnellement au nombre des actions détenues par les actionnaires..$^{85}$

Après la vente du Richelieu et avec le Jacques Cartier destiné uniquement au commerce du Saint-Laurent, une ère prenait fin dans l'histoire de la Compagnie du Richelieu dont le nom était devenu inapproprié. La transition du Richelieu au SaintLaurent, déjà commencée en 1850-1851, était maintenant accomplie. Des hommes d'affaires montréalais, tels que Marchand, Lamothe, Boulanger et Hudon, formaient depuis 1851, une ma-

82 Ibid.

83 Archives Publiques du Canada, R.G. 4, C1, Provincial Secretary's Correspondence, CCLXXVIII $(1850): 1225$.

84 Procès-verbaux du 15 fév. 1853.

85 Ibid., du 20 mars 1853. 
jorité écrasante parmi les directeurs de la Compagnie qui prenait de plus en plus le caractère d'une entreprise localisée à Montréal et propriété de Montréalais. Et tandis que les Monttréalais concentraient la direction entre leurs mains, la viabilité économique de la Compagnie dépendait de la métropole où d'autres entrepreneurs parachevaient le "St. Lawrence and Atlantic Railway". Ainsi, vers 1853, Montréal avait entièrement attiré la Compagnie qui, par la suite, se spécialisa exclusivement dans le transport sur le Saint-Laurent. Pendant les trois années suivantes la Compagnie du Richelieu démontra non seulement qu'elle pouvait résister à la concurrence rigoureuse de quatre autres compagnies de bateaux à vapeur qui se disputaient le transport entre Montréal et Trois-Rivières, mais qu'elle avait aussi le "leadership" nécessaire pour s'unir à trois de ces firmes rivales, la Compagnie du lac Saint-Pierre, la Compagnie du Cultivateur et la Compagnie de Traverse de Laprairie, et former, en mars 1856, la Compagnie de Navigation de Montréal à Trois-Rivières ${ }^{86}$.

La nouvelle Compagnie aurait trois bateaux à vapeur pour le commerce entre Montréal et Trois-Rivières, ainsi que deux vaisseaux neufs encore sur cales au chantier Cantin de Montréal, pour le trajet de Montréal à Québec. ${ }^{87}$ A la veille de la saison maritime de 1856, dix ans après s'être lancée sur le Richelieu et le Saint-Laurent, la Compagnie s'était transformée en un grand syndicat créé pour le commerce du transport entre les centres majeurs de Montréal, de Trois-Rivières et de Québec. Ainsi l'année 1853 marqua à la fois la fin d'une période de transition et les débuts d'une étape nouvelle dans l'histoire de la Compagnie du Richelieu qui pourrait faire l'objet d'un long développement.

\section{$\mathrm{V}$}

Un grand nombre, sinon la plupart des bateaux à vapeur le long du Richelieu et du Saint-Laurent appartenaient à des groupes ou à des particuliers canadiens-français. La presque totalité des actions de la Compagnie du Richelieu, ainsi que la propriété des vaisseaux que concurrença la Compagnie appartenaient à des marchands canadiens-français dont plusieurs venaient des rives du Saint-Laurent et de celles du Richelieu, et, en nombre croissant avec les années, de Montréal.

L'étude de cette forme d'entreprise maritime montre que des Canadiens français, en particulier de Montréal, réussirent à se

86 Ibid., du 31 mars 1856.

87 Ibid. 
maintenir à la tête de la Compagnie du Richelieu, l'une des firmes les plus considérables de ce genre. Elle contribue également et surtout à la ré-interprétation de l'histoire économique du Québec au cours de cette période. Il importe de souligner que plusieurs entreprises canadiennes-françaises, dont notamment la Compagnie du Richelieu, se distinguèrent par leur intérêt et leur détermination dans la recherche de profits (ce que l'on qualifie parfois "d'éthique protestante") qui leur permirent de survivre à une concurrence acharnée.

Ce sujet comporte un intérêt tout particulier car il invite à réexaminer quelques-unes des généralisations que l'on a faites sur le manque d'esprit d'entreprise commerciale des Canadiens français. Fernand Ouellet a affirmé que les Canadiens français n'ont pas su relever le défi de l'économie moderne commerciale dès ses débuts, à la fin du XVIII ${ }^{\mathrm{e}}$ siècle, alors que dans le commerce des fourrures, par exemple, "l'entreprise canadienne-française n'a pas été suffisamment sensible à l'appel de la conjoncture... [des] exigences du temps..." 88 Etienne Parent, dans les années 1840, attribuait principalement à leur "fol engouement pour les professions libérales" 89 , l'échec des Canadiens français à jouer pleinement leur rôle dans les affaires. Le sociologue Norman Taylor a soutenu récemment que la résistance aux changements ou l'inadaptation aux variations de conjoncture économique caractérise encore de nos jours le comportement de nombreux hommes d'affaires canadiens-français. ${ }^{90}$ Ce sujet nécessiterait une recherche beaucoup plus poussée, particulièrement dans le domaine d'entreprises telles que la Compagnie du Richelieu et dans le monde des affaires de la petite et moyenne bourgeoisie. La découverte d'autres petites entreprises du genre ayant connu un succès plus considérable que ce qui apparaissait à

88 Ouellet, op. cit., 104.

89 Ramsay Cook, ed., French-Canadian Nationalism, An Anthology (Toronto, 1969), 85. Trente ans plus tard, pourtant, Laurent-Olivier David a cru trouver cinq raisons majeures pour expliquer le retard économique et la lenteur des progrès de l'esprit d'entreprise au Canada français: la psychologie des peuples (l'esprit latin vs le matérialisme anglo-saxon), des facteurs historiques, l'absence d'une éducation pratique (la principale cause selon L.-O. David), l'ignorance et l'avarice des hommes d'affaires canadiensfrançais, et, finalement, le mauvais emploi des capitaux par une spéculation improductive. Yves-F. Zoltvany, "Laurent-Olivier David et l'infériorité économique des Canadiens français", Recherches sociographiques, X (1969): 426-442.

$90 \mathrm{~N}$. Taylor, "The French-Canadian Industrial Entrepreneur and his Social Environment", dans Rioux et Martin, éd., French-Canadian Society (Toronto, 1965), I: '271-295, passim. Cf. William Ryan, The Clergy and the Economic Growth of Quebec, 1896-1914 (Quebec, 1966), passim. 
prime abord entraînerait forcément une revision de ces généralisations. En poussant davantage la recherche, on pourrait peut-être déterminer dans quelle mesure les entrepreneurs canadiens-français, en s'associant ou en investissant leurs capitaux, furent affectés par ce que Alfred Dubuc affirme avoir été la nécessité "de participer aux valeurs et aux comportements propres au groupe dominant des entrepreneurs" ${ }^{91}$. A partir des preuves ici présentées, nous croyons pouvoir conclure que les Canadiens français furent, sur le plan commercial, aussi agressifs et aussi souples que d'autres dans la région de Montréal, et que certains d'entre eux se révélèrent tout à fait aptes à relever avec succès les défis du transport maritime au milieu du XIX $\mathrm{X}^{\mathbf{e}}$ siècle.

Traduction

91 Alfred Dubuc, "Problems in the Study of the Stratification of the Canadian Society from 1760 to 1840 ", Canadian Historical Association, Report (1965), 23. Le texte de cette communication a été publié en français sous le titre: "Les classes sociales au Canada", dans Annales, E.S.C., 22e année, no 4 (juillet-août 1967): 829-844. 